\title{
Die Zeitschrift für Orthopädie - Beständigkeit und
}

F. U. Niethard

Wandel

Die Zeitschrift für Orthopädie hat ein verändertes Gesicht. Seit einem Jahr erscheint sie unverkennbar als Publikation des Thieme-Verlages in blauem Gewand. Nun hat sich auch das Innere der Zeitschrift geändert: Das Layout ist modernisiert. Schriftleitung und Verlag hoffen, damit auch den stets steigenden Ansprüche an eine anschauliche und übersichtliche Präsentation der Publikationen gerecht zu werden.

Veränderungen gibt es auch im Kreise der Herausgeber und der Gutachter. Herr Prof. Dr. Hackenbroch ist mit seiner Emeretierung nach 14jähriger Tätigkeit als Herausgeber der Zeitschrift für Orthopädie ausgeschieden. Die Schiftleitung, die Mitherausgeber und der Verlag danken Herrn Prof. Hackenbroch für seine engagierte Mitarbeit, die den Stellenwert der Zeitschrift für Orthopädie als bestes nicht englischsprachiges Publikationsorgan für Orthopädie gefestigt hat.

Als „Maß“ für den Stellenwert einer wissenschaftlichen Zeitschrift, so auch der Zeitschrift für Orthopädie, gilt der ImpactFactor. Dass dieser - vom Institute for Scientific Information (ISI) in Philadelphia jährlich neu festgelegt - umstritten ist, wurde in dieser Zeitschrift wiederholt diskutiert (Niethard 1996, 2001) $[4,5]$. Dessen ungeachtet ist er bisher der einzige Maßstab, um die Akzeptanz einer Zeitschrift auf dem wissenschaftlichen Feld zu quantifizieren. Auch im Jahr 2000 ist die Zeitschrift für Orthopädie mit einem Impact-Factor von 0,446 nach wie vor bestes, nicht englischsprachiges Publikationsorgan in der Orthopädie (Tab.1). Dies ist um so höher zu bewerten, als die deutschsprachigen Publikationsorgane im angelsächsischen Schrifttum offenbar gering beachtet und geachtet werden. Füeßl (2000) [1] weist darauf hin, dass es in der englischsprachigen Literatur von orthographischen Fehlern und sinnentstellenden Formulierungen bei Literaturzitaten aus deutschsprachigen Publikationsorganen geradezu „strotzt“. Kein Journal würde das bei eng- lischen Zitaten tolerieren. „Mark Twains maliziöse Anmerkungen „the awful german language“ scheint in vielen englischsprachigen Köpfen zu stecken“ (Füeßl 2000) [1]. Zu Recht weist der Autor darauf hin, dass durch diese einseitige Sicht, die für die heutige Entwicklung von Leitlinien wichtigen Metaanalysen, z. B. der Cochrane Library, verfälscht werden.

Aus diesem Grund wurde in dieser Zeitschrift bereits die Forderung nach einem „europäischen Impact-Factor“ aufgestellt (Niethard, 2001) [5]. Nun liegt ein erster Entwurf für einen solchen „Euro-Faktor“ vor (Hofbauer, 2002) [2]. Die von Hofbauer iniziierte Arbeitsgruppe hat das Dilemma erkannt, dass europäische Arbeiten kaum in amerikanischen Journalen veröffentlicht werden. Trotz der Sprachgrenzen (Sprach-Bias, Füeßl, 2000) [1] wird in Europa zunehmend der Impact-Factor als alleiniger Bewertungsmaßstab für die Leistungsfähigkeit von Journalen aber auch von Fakultäten und ganzen Universitäten herangezogen, was nicht einmal in den USA der Fall ist. Aus diesem Grund ist der Entwurf eines europäischen Impact-Factors ein mutiger Schritt in die richtige Richtung. Auch bei diesem „Euro-Faktor“ ist die Zeitschrift für Orthopädie gut und als beste nicht englischsprachige Zeitschrift im europäischen Raum platziert (Tab.2). Noch sind offenbar nicht alle Journale des europäischen Raumes erfasst (z.B. Revue orthopédique). Fünf der bislang gelisteten orthopädischen Zeitschriften sind englischsprachig. Dies ist Ausdruck des Circulus vitiosus, nachdem ein geringer Impact-Factor die nicht englischsprachigen Autoren dennoch in die angelsächsische Literatur treibt (Köbberling 2000) [3]. Englisch wird die Sprache der internationalen Wissenschaft bleiben. Der europäische Impact-Factor aber schafft die Möglichkeiten, die Vorgaben des „europäischen Publikationsmarktes“ besser zu berücksichtigen. Mit Modifikationen ausgestattet wünschen wir ihm viel Erfolg. 
Tab. 1 Impact-Factor

\begin{tabular}{|c|c|c|}
\hline Rang & Journal & Impact-Factor \\
\hline 1 & JORTHOPAED RES & 2.233 \\
\hline 2 & J BONE JOINT SURG & 2.222 \\
\hline 3 & OSTEOARTHR & 2.080 \\
\hline 4 & SPINE & 1.843 \\
\hline 5 & J BONE JOINT SURG BR & 1.612 \\
\hline 6 & J ORTHOP SPORT PHYS & 1.424 \\
\hline 7 & CLIN J SPORT MED & 1.255 \\
\hline 8 & PHYS THER & 1.222 \\
\hline 9 & ARTHROSCOPY & 1.217 \\
\hline 10 & CLIN ORTHOP RELAT R & 1.182 \\
\hline 11 & CLIN BIOMECH & 1.056 \\
\hline 12 & J ARTHROPLASTY & 0.978 \\
\hline 13 & ACTA ORTHOP SCAND & 0.973 \\
\hline 13 & J SHOULDER ELB SURG & 0.973 \\
\hline 15 & GAIT POSTURE & 0.955 \\
\hline 16 & CONNECT TISSUE RES & 0.952 \\
\hline 17 & ORTHOP CLIN N AM & 0.874 \\
\hline 18 & J ORTHOP TRAUMA & 0.843 \\
\hline 19 & J SPINAL DISORD & 0.816 \\
\hline 20 & J HAND SURG-AM & 0.795 \\
\hline 21 & J PEDIATR ORTHOPED & 0.636 \\
\hline 22 & HAND CLIN & 0.571 \\
\hline 23 & ARCH ORTHOP TRAUM SU & 0.507 \\
\hline 24 & J HAND SURG-BRIT EUR & 0.495 \\
\hline 25 & FOOT ANKLE INT & 0.493 \\
\hline 26 & ISOKINET EXERC SCI & 0.476 \\
\hline 27 & ORTHOPEDICS & 0.472 \\
\hline 28 & Z ORTHOP GRENZGEB & 0.446 \\
\hline 29 & INT ORTHOP & 0.368 \\
\hline 30 & ORTHOPADE & 0.364 \\
\hline 31 & NEURO-ORTHOPEDICS & 0.273 \\
\hline 32 & J BACK MUSCULOSKELET & 0.261 \\
\hline 33 & KNEE & 0.255 \\
\hline 34 & J PEDIATR ORTHOP B & 0.217 \\
\hline 35 & REV CHIR ORTHOP & 0.212 \\
\hline 36 & J AM PODIAT MED ASSN & 0.189 \\
\hline 37 & PROSTHET ORTHOT INT & 0.119 \\
\hline 38 & JNMS-J NEUROMUSC SYS & 0.103 \\
\hline 39 & CURR ORTHOPAED & 0.099 \\
\hline
\end{tabular}

Tab. 2: Euro-Factor (EF) ${ }^{\mathrm{TM}}$

\begin{tabular}{lll}
\hline 1 & Acta Orthop Scand & 6.78 \\
\hline 2 & Osteoporosis Int & 4.86 \\
\hline 3 & Arch Orthop Traum Surg & 3.01 \\
\hline 4 & Int Orthop & 2.67 \\
\hline 5 & Z Orthop & 2.50 \\
\hline 7 & Osteoarthr Cartilage & 2.15 \\
\hline
\end{tabular}

\section{Literatur}

${ }^{1}$ Füeßl HS. Die Zukunft der deutschen medizinischen Journale. Dtsch Med Wschr 2000; 125: 1103 - 1105

${ }^{2}$ Hofbauer R. Euro-Factor $(\mathrm{EF})^{\mathrm{TM}}$ - The European Journal Quality Factor. Vicer Publishing, Wien: 2001

${ }^{3}$ Köbberling J. Qualität deutscher Journale. Dtsch Med Wschr 2000; 125: $1106-1108$

${ }^{4}$ Niethard FU. Im Pakt mit dem Impact-Faktor. Z Orthop 1996; 134: $293-294$

${ }^{5}$ Niethard FU. Die Zeitschrift für Orthopädie - Online. Z Orthop 2001; 139: $1-1$ 